\title{
Careful Reading versus Expeditious Reading: Investigating the Construct Validity of a Multiple-choice Reading Test
}

\author{
Godefroid B. Katalayi \\ University of the Western Cape, South Africa \\ Sivakumar Sivasubramaniam \\ University of the Western Cape, South Africa
}

\begin{abstract}
The paper investigates the validity of a multiple-choice reading test using Weir and Khalifa's (2008) model of reading. Fifty (50) multiple-choice reading items taken from the 2011 English state examination were administered to 496 Grade 12 secondary school students. A concurrent strategies questionnaire was used to elicit information on participants' use of strategies during test writing. The results indicate that there are more items that target careful reading than those that target expeditious reading. This is to suggest that the ESE tasks appear to be inappropriate and may need some modifications to more closely reflect the actual test context.
\end{abstract}

Index Terms - construct validity, careful reading, expeditious reading, reading construct, reading context, task complexity

\section{INTRODUCTION}

In this article, we apply Weir and Khalifa's (2008a) model of reading types to validate the English state examination, a subtest of the national test administered to high school finalist students in the Democratic Republic of the Congo for certification.

Weir and Khalifa's model conceptualizes reading as a multi-componential construct where reading can be broken down into underlying skill or strategy components for the purpose of both teaching and testing (Weir \& Khalifa, 2008a; Khalifa \& Weir, 2009). Consistent with this conceptualization, reading construct needs to be described by looking at how readers engage constellations of strategies to construct text meaning and complete test tasks.

In their framework, Weir and Khalifa conceptualize reading construct on a four-matrix cell with careful reading and expeditious reading that can be conducted at both global level and local level. Such a conceptualization of reading is a development in the reading theory because many existing reading models (for instance Bernhardt, 1991; Hoover \& Tunmer, 1993; Rayner \& Pollatsek, 1989) are nearly premised on a careful reading model and do not take sufficient account of the different purposes of reading. Yet, careful reading alone may be an inadequate construct for students as expeditious reading may tell us how readers can cope with other common life reading behaviours such as skimming or search reading (Weir \& Khalifa, 2008a; Weir et al., 2008a).

Careful reading refers to different operations where the reader attempts to extract complete meanings within or beyond sentences right up to the level of the entire text so as to construct the text macrostructure (Weir \& Khalifa, 2008a; Khalifa \& Weir, 2009). Careful reading is a construct that is operationalized through the identification of lexis, the understanding of syntax, the seeking of an accurate comprehension of explicit meaning and the making of inferences (Weir \& Khalifa, 2008a; Khalifa \& Weir, 2009). These tasks may take place at a local level (understanding lexis, identifying grammar) or at global level (seeking an accurate comprehension of explicit meaning and making inferences). Careful reading at the local level involves processing a text until the basic meaning of a proposition is established whereas careful reading at the global level involves processing the text until its macro-structure is built.

Expeditious reading involves a quick, selective and efficient reading to access needed text information (Weir \& Khalifa, 2008a; Urquhart \& Weir, 1998). In expeditious reading, the linearity of text is not necessarily followed as the reader attempts to sample the text in order to extract pieces of information necessary to answer specific test items (Urquhart \& Weir, 1998).

Expeditious reading is a construct that encompasses a range of reading types (Urquhart \& Weir, 1998), micro-skills (Munby, 1978), skills (Levine et al. 2000), abilities (Enright et al., 2000; Cohen \& Upton, 2007) or strategies (Purpura, 1998). These overlapping concepts demonstrate some confusion in existing literature to distinguish between skills and strategies, and some researchers use them as interchangeable concepts (for example Grabe, 1999). However, the simplest distinction between skill and strategy may be that skills are text-driven, largely subconscious linguistic processes involved in reading (Weir et al. 2000) while strategies are purposeful and conscious responses to local 
problems in a text (Mebarki, 2012), that is, some ways readers employ to get round difficulties encountered while reading (Urquhart \& Weir, 1998).

Expeditious reading can be conducted at global level (search reading and skimming) or at local level (scanning). In search reading, the reader processes the text in order to locate only text information necessary for answering specific test questions (Urquhart \& Weir, 1998); a process that resembles to sampling the text to extract needed pieces of information. On the other hand, in skimming, the reader processes the text in order to obtain the gist; trying to avoid details through a thorough selection of as few text details as possible (Weir \& Khalifa, 2008a; Levine et al. 2000; Weir 2005). In scanning, the reader processes the text selectively in order to achieve specific goals; attempting to dismiss any part of the text that does not contain useful information for answering a specific test item (Weir and Khalifa, 2008a).

\section{Reading construct and reading context as core elements in validating reading tests}

In validating reading tests, the reading construct cannot be examined outside the appropriate context of reading activity; a hypothesis that suggests an interaction between test construct and test context (Weir, 2005). In keeping with this hypothesis, examinees' capacity to deploy appropriate strategies to read the text and complete the test tasks also depends on the extent to which the test tasks are appropriate to examinees' characteristics (their skills, motivation for reading, experience with the test tasks, attitude towards the test), the conditions under which reading instruction takes place (classroom size, reading resources, quality and content of the curriculum), as well as the conditions under which testing occurs.

Since the DR Congo is a French speaking country and English is learnt as a foreign language, its use is principally limited to classroom context; suggesting students' limited exposure to English. An investigation of the DR Congo English state examination context by Katalayi (2011) reveals the following: (1) students' reading at school is very restricted as the time allocated for learning English is one to five hours per week depending on the subject areas; (2) reading at home is almost inexistent due to lack of reading materials; where these exist, their contents are sometimes irrelevant to students' needs; (3) many students are not motivated in the English course as they do not regularly attend English classes. This may be a result of their perception of English as a difficult subject; (4) some students have a negative attitude towards the ESE as they sometimes believe that this test is a way of failing them on the national test; (5) although those who teach English are generally formally qualified to teach it, there are no in-service trainings as well as continuous trainings to update them with new developments in teaching; (6) although the multiple-choice method is used with the English state examination, the majority of teachers use the open questions as classroom assessment method on the ground that the multiple-choice method is difficult to construct.

In this article, we intended to determine the construct validity of the ESE through an investigation of task complexity of the four reading types. In order to achieve this objective, the main aims were:

- Determine task complexity of different reading types;

- Evaluate the extent to which the ESE tasks are appropriate to the examinees.

\section{BACKGROUND}

In adopting Weir and Khalifa's model of reading comprehension, test questions may be generally classified on the basis of whether the examinee has to read the text carefully or expeditiously at global or local level in order to answer individual test questions (Weir \& Khalifa, 2008b, Weir, Hawkey, Green \& Devi, 2008; Weir, Hawkey, Green, Unaldi \& Devi, 2008; and Sarojani \& Krishnan, 2011). This is to suggest that, theoretically, in answering reading questions, the examinee attempts to identify the type of questions and the relevant information source (van Steensel, Oostdam \& van Gelderen, 2012; Cerdan et al., 2009; Rouet et al., 2001).

Research shows differences in the use of strategies between test items that require examinees to read at global level and those that require them to read at local level. Rouet et al. (2001) reported that 'low-level questions' required examinees to read the text at local level by focusing on text micro-propositions and quickly searching for text information through a browsing of some parts of the text. On the contrary, 'high-level questions' required examinees to read the text at global level by focusing on text macro-propositions and by attempting to generate more connections between their knowledge and text information.

Researchers have generally tended to classify local test items as those that are 'easy' as such items involve retrieving specific pieces of information located in specific parts of the text (van Steensel, Oostdam \& van Gelderen, 2012; OECD, 2003; Song, 2008; Rouet et al., 2001). Such text information is generally a verbatim, or requires minor change as the case of a test item that requests examinees to find the synonym or antonym (OECD, 2003; Cerdan et al., 2009). On the other hand, researchers have generally tended to classify global test items as 'difficult' items as such items involve text global comprehension as the case of test items that require the examinees to find a title that best suits the text (OECD, 2003; Song, 2008) or to make inferences on the basis of information not explicitly stated in the text (van Steensel, Oostdam \& van Gelderen, 2012; Weir \& Khalifa, 2008b; Weir, Hawkey, Green \& Devi, 2008; Weir, Hawkey, Green, Unaldi \& Devi, 2008; and Sarojani \& Krishnan, 2011). In many cases, most of these researchers distinguish test questions that are half-way between these two categories. These questions are 'moderate' questions and they tap to examinees' comprehension of both underlying relationships between local level text information and global level text information (van Steensel, Oostdam \& van Gelderen, 2012; Weir \& Khalifa, 2008b). 
Validation studies that used Weir and Khalifa's (2008a) framework generally sought to determine whether the test mainly includes tasks that require the examinees to read the text carefully or expeditiously. In a study aimed to determine the variety and complexity of the reading types required by the test tasks in the IELT, Weir et al. (2008a) found that $77 \%$ of strategies used by participants to complete test tasks tapped to careful reading while only $23 \%$ of strategies tapped to expeditious reading. The finding suggests that the IELT Reading Module test may need possible modification to more closely represent the academic reading construct of university students through texts and tasks that test more extensively students' expeditious reading skills. This finding was replicated in a study by Weir et al. (2008b) when they found that the major focus of the IELTS test papers appeared to be on careful reading although most participants used expeditious reading skills and strategies in answering test questions; a finding that suggests that expeditious reading may be a general reading strategy as some participants may decide to read the text quickly and selectively before approaching the test questions. This preponderance of careful reading strategies over expeditious reading strategies was also reported by Sarojani and Krishnan (2011)'s replicative study on IELT when he found that the majority of IELT test items tested careful reading although the majority of participants perceived expeditious reading skills as more relevant and appropriate to their academic reading activity.

The preponderance of careful reading tasks over expeditious reading tasks is generally featured through the scarcity of items that target skimming and search reading skills. Weir et al. (2000) who studied samples of EAP reading tests such as IELTS found that skimming rarely featured in items in this test, and when it did, it was realized in only a single item asking "what is the main idea of the passage?". A similar finding was reported by Weir and Khalifa (2008b) for Main Suite when they found that this test did not include enough tasks that would have reflected examinees' capacity to skim the text.

Since expeditious reading involves quick and selective reading, it may not be easy to discriminate skills that are involved while reading the text selectively to extract specific pieces of information. In their investigation on the relationship between the academic reading construct as measured by IELTS and the reading experiences of students in their first year of study at university, Weir et al. (2008a) reported that the three expeditious reading strategies of skimming, search reading and scanning involved some overlapping processes and actions; suggesting a confusion some researchers might have in labeling these three strategies. Nonetheless, if skimming takes place at global level only, and scanning takes place at local level only, search reading may take place at both global and local levels.

Since scanning involves reading selectively at local word level to achieve very specific reading goals, research generally associates items that require examinees to scan the text to easiest types of reading items (Weir \& Khalifa, 2008b).

\section{METHOD}

A process-oriented approach to examining the reading construct needs a method that enables to comment on the actual reading process itself. Hence, protocol analysis can provide light for understanding the processes examinees engage to comprehend the text and complete test tasks. This method has become popular as a methodology to uncover psychological processes that a person goes through while performing a task (Faerch \& Kasper, 1987; Ericsson \& Simon, 1993). It is based on the hypothesis that since people have "privileged access to their experiences" (Ericsson \& Simon, 1993: xii), and that the information in their verbal reports is trustworthy (Park, 2009), it is possible to verbalize their thoughts in a way that does not alter the sequence of thought that mediate the completion of the task (Ericsson \& Simon, 1993). Protocol analysis methodology has gained a strong ground in validating reading tests (cf. Rupp, Ferne, \& Choi, 2006; Sasaki, 2000; Yamashita, 2003; Nevo, 1989; Weir \& Khalifa, 2008b; for example).

Many validation studies of the reading construct (Weir 2005; Hirano, 2008; Cohen \& Upton, 2007; for instance) highlight the importance of establishing as clearly as possible what examinees are actually doing while completing test tasks on the ground that what a task actually tests is what is central to validity. Therefore, an investigation of examinees' strategies can provide understanding as to how they interact with the text and how their selection of strategies influences their comprehension of the text and their performance on the test.

In conducting protocol analysis, the underlying assumption is that the way examinees search for text information, evaluate item alternatives, and choose the best option can be registered through their verbalizations and later analyzed to discover their decision processes and patterns (Kuusela \& Paul, 2000). This is why the data were collected during the task completion process in order to ensure close connection between thinking and verbal reports (Simon, 1993; Ericsson \& Simon, 1993).

\section{A. Sample, Instrument and Materials}

The participants in the present study were 496 Grade 12 secondary school students who were in the last grade of secondary school and who were preparing for the national test in order to get a national certificate.

A concurrent strategies questionnaire adapted from Weir and Khalifa's (2008a) questionnaire was used to elicit information on participants' use of strategies during test writing. In this questionnaire, participants were instructed to select from a list of strategies (cf. Appendix), individual strategies they have used to answer each test question immediately after they had answered that individual test question. 
The test material consisted of 50 multiple-choice reading test items taken from the 2011 edition test. This test was administered to the 496 participants under normal testing conditions.

\section{B. Procedure}

Participants were told that the test was accompanied with a questionnaire that was to be completed while taking the test. Participants were instructed fill out the questionnaire each time immediately after answering an individual test question. They were reminded that they had to carefully go through the list of suggested strategies in the questionnaire and check all strategies they remembered they actually used to find the answer to each individual question.

\section{Analysis}

The task complexity of the four reading types was determined through an examination of strategies used by participants to answer individual test questions (see Appendix for strategies coding). This was triangulated with an investigation of item difficulty of different test questions included in each reading types.

In order to examine the strategies used by participants, the frequency of strategies was computed and Cohen and Upton's (2007) label was used. Strategies used by more than $75 \%$ of participants in answering an individual test question were labeled 'high' $(\mathrm{H})$ frequency; those used by 50 to $74 \%$ of participants were labeled 'moderate' (M) frequency, whereas those used by less than $50 \%$ of participants were labeled 'low' (F) frequency.

The item difficulty of individual test items is a straightforward statistics that consists in taking the number of candidates who got an item right and divide this number by the total number of candidates who answered that item. From the results of item difficulty per item, the mean item difficulty was computed for different reading skills included in each of the four reading types. In order to show any significance variance that might occur between item difficulty indexes of different items included in the same category, the standard deviation was computed. This is a statistics that aims to reflect the dispersion (variance) observed among item difficulty index of items grouped in the same category. Values closer to 1 suggest that the item is easy; values closer to .0 suggest that the item is difficult whereas values that cluster around .5 suggest that the item is of moderate difficulty.

\section{Results}

\section{A. Task Complexity of Careful Reading at Global Level Test Questions}

The information presented in Table 1 suggests that in order to answer test questions that required participants to carefully read the text at global level, the main strategy used by participants was 'read the whole text carefully' (S1). In some instances, participants reread the text rapidly (S2) in order to build its gist. If the question requested them to establish text or paragraph global comprehension, they read a specific paragraph of the text carefully (S3) and rapidly (S4) in order to build the gist of that paragraph. The mean item difficulty of .4 substantiates that these questions were difficult for participants.

On the other hand, if the test question required their skill to establish accurate comprehension of explicitly stated main ideas and supporting details across sentences, participants mainly read the target paragraph carefully (S3) with the aim to comprehend all supporting paragraph details as elaborated across different sentences. For some participants, they first read the whole text rapidly (S2) in order to construct its representation before focusing on the target paragraph and read it carefully (S3). The mean item difficulty index of .3 suggests that the three test items that required participants to establish accurate comprehension of explicitly stated main ideas and details across sentences were difficult.

In order to answer textually implicit questions that require their skills to combine various pieces of information across the different sentences in the text and make inferences on the basis of these pieces of information, participants combined careful reading (S1) and expeditious reading (S2) of the whole text; and for some test items whose pieces of information were to be found across sentences within a single specific paragraph, participants carefully read that specific paragraph (S3). Besides this careful and expeditious reading of the whole text or a specific portion of the text, some participants had to draw some conclusions based on what the text/paragraph implied (S7). The mean item difficulty index of .5 suggests that the inferencing questions were of moderate degree. 
TABLE 1:

ITEM DIFFICULTY AND FREQUENCY OF READING AND TEST TAKING STRATEGIES FOR THE FOUR READING TYPES

\begin{tabular}{|c|c|c|c|c|c|c|c|c|c|c|c|c|c|c|c|}
\hline \multirow{2}{*}{$\begin{array}{l}\text { Reading types and reading } \\
\text { skills }\end{array}$} & \multirow{2}{*}{$\begin{array}{l}\mathrm{N} \\
\text { item }\end{array}$} & \multirow[t]{2}{*}{ ID } & \multicolumn{8}{|c|}{ Reading strategies } & \multicolumn{5}{|c|}{ Test taking strategies } \\
\hline & & & S1 & $\mathrm{S} 2$ & S3 & S4 & S5 & S6 & S7 & S8 & S9 & $\mathrm{S} 10$ & S11 & $\mathrm{S} 12$ & S13 \\
\hline \multicolumn{16}{|l|}{ Careful reading at global level } \\
\hline $\begin{array}{l}\text { Establishing text/paragraph } \\
\text { global comprehension }\end{array}$ & 6 & .4 & $\mathrm{H}$ & $\mathrm{M}$ & $\mathrm{H}$ & $\mathrm{M}$ & - & - & - & - & $\mathrm{M}$ & $\mathrm{L}$ & - & $\mathrm{M}$ & $\mathrm{L}$ \\
\hline $\begin{array}{l}\text { Establishing accurate } \\
\text { comprehension of explicitly } \\
\text { stated main ideas and } \\
\text { supporting details across } \\
\text { sentences }\end{array}$ & 3 & .3 & $\mathrm{M}$ & $\mathrm{M}$ & $\mathrm{H}$ & $\mathrm{L}$ & - & - & $\mathrm{L}$ & - & $\mathrm{H}$ & $\mathrm{L}$ & - & $\mathrm{L}$ & $\mathrm{L}$ \\
\hline $\begin{array}{l}\text { Making text/paragraph } \\
\text { inferences }\end{array}$ & 7 & .5 & $\mathrm{M}$ & $\mathrm{M}$ & $\mathrm{L}$ & $\mathrm{L}$ & & & $\mathrm{L}$ & & $\mathrm{L}$ & $\mathrm{L}$ & & $\mathrm{L}$ & \\
\hline \multicolumn{16}{|l|}{ Careful reading at local level } \\
\hline understanding lexis & 9 & .5 & $\mathrm{~L}$ & & & & $\mathrm{~L}$ & $\mathrm{H}$ & & $\mathrm{L}$ & $\mathrm{M}$ & $\mathrm{L}$ & & $\mathrm{M}$ & $\mathrm{L}$ \\
\hline Understanding syntax & 7 & .6 & & $\mathrm{~L}$ & $\mathrm{~L}$ & & $\mathrm{H}$ & $\mathrm{L}$ & & $\mathrm{L}$ & $\mathrm{L}$ & $\mathrm{H}$ & $\mathrm{M}$ & $\mathrm{M}$ & \\
\hline \multicolumn{16}{|c|}{ Expeditious reading at global level } \\
\hline $\begin{array}{l}\text { Skimming the text/paragraph } \\
\text { to locate needed information }\end{array}$ & 5 & .7 & $\mathrm{~L}$ & $\mathrm{H}$ & $\mathrm{L}$ & $\mathrm{L}$ & & & $\mathrm{L}$ & & & $\mathrm{H}$ & & $\mathrm{L}$ & \\
\hline $\begin{array}{l}\text { Search reading to quickly } \\
\text { locate explicit pieces of } \\
\text { information scattered through } \\
\text { the whole text/paragraph }\end{array}$ & 7 & .4 & $\mathrm{~L}$ & $\mathrm{H}$ & $\mathrm{L}$ & & $\mathrm{L}$ & & & & $\mathrm{L}$ & $\mathrm{L}$ & & $\mathrm{M}$ & $\mathrm{L}$ \\
\hline \multicolumn{16}{|c|}{ Expeditious reading at local level } \\
\hline $\begin{array}{l}\text { Scanning to locate explicit } \\
\text { information located in a } \\
\text { specific part of the } \\
\text { sentence/paragraph }\end{array}$ & 6 & .6 & & & $\mathrm{~L}$ & & $\mathrm{~L}$ & $\mathrm{~L}$ & & $\mathrm{M}$ & & & $\mathrm{L}$ & $\mathrm{M}$ & \\
\hline
\end{tabular}

\section{B. Task Complexity of Careful Reading at Local Level Test Questions}

The information presented in Table 1 suggests that in order to answer test questions that required participants to carefully read the text at local level, two main strategies were used: either participants used their knowledge of grammar (S5) for items requiring their understanding of grammar or they used their knowledge of vocabulary (S6) for items requesting their understanding of vocabulary. For some participants, they had first to carefully and/or quickly read the whole text before making their choice (S1, S2). This reading of the whole text may aim to enable them grasp the text context that might provide context clues to figure out the word meanings or the context of use of grammar structures. Since grammar structures or vocabulary words were situated within single sentences, participants had first to look for parts of the text that the writer had recommended (S8). Data signal that for some vocabulary items, participants had also to resort to their knowledge of grammar (S5) and that for some grammar items, they had also to resort to their knowledge of vocabulary. The mean item difficulty for the nine test questions that targeted examinees' understanding of lexis (.5) and the seven test questions that targeted examinees' understanding of grammar (.6) suggests that these types of questions were of moderate difficulty.

TABLE 2:

COMPARING TEST FOCUS AND TASK DIFFICULTY BETWEEN CAREFUL READING AND EXPEDITIOUS READING

\begin{tabular}{|c|c|c|c|c|c|}
\hline \multirow{2}{*}{\multicolumn{2}{|c|}{$\begin{array}{l}\text { Careful reading versus expeditious reading: Item } \\
\text { frequency and mean item difficulty }\end{array}$}} & \multicolumn{2}{|c|}{ Item frequency } & \multirow{2}{*}{$\begin{array}{l}\text { Mean item } \\
\text { difficulty }\end{array}$} & \multirow[t]{2}{*}{ SD } \\
\hline & & $\mathrm{N}=50$ & $\%$ & & \\
\hline 1 & Careful reading & 32 & 64 & .5 & 0.18 \\
\hline 2 & Expeditious reading & 18 & 36 & .6 & 0.16 \\
\hline
\end{tabular}

TABLE 3:

COMPARING TEST FOCUS AND TASK DIFFICULTY BETWEEN READING AT GLOBAL LEVEL AND READING AT LOCAL LEVEL

\begin{tabular}{|c|c|c|c|c|c|}
\hline \multirow{2}{*}{\multicolumn{2}{|c|}{$\begin{array}{l}\text { Reading at global level versus reading at local } \\
\text { level: Item frequency and mean item difficulty }\end{array}$}} & \multicolumn{2}{|c|}{ Item frequency } & \multirow{2}{*}{$\begin{array}{l}\text { Mean item } \\
\text { difficulty }\end{array}$} & \multirow[t]{2}{*}{ SD } \\
\hline & & $\mathrm{N}=50$ & $\%$ & & \\
\hline 1 & Reading at global level & 28 & 56 & .5 & 0.15 \\
\hline 2 & Reading at local level & 22 & 44 & .6 & 0.19 \\
\hline
\end{tabular}

\section{Task Complexity of Expeditious Reading at Global Level Test Questions}

The information offered in Table 1 signals that, in order to answer test questions that required examinees' skill to skim the text/paragraph to locate needed information, the most frequently used strategy was 'read the whole passage rapidly (S2)' in order to quickly locate needed information. If the needed information was located in a specific paragraph, examinees could also read that specific paragraph rapidly (S4) and sometimes also carefully (S3). Since all these test questions presented a stem with information not explicitly stated in the text (for example "In which part of the text can we find the idea that 'one person can decide on the place where to construct a city?"') some examinees had to draw conclusion on the basis of what the text/paragraph implied (S7). The mean item difficulty (.7) suggests that this type of items was easy. 
On the other hand, in order to answer test items that required examinees to quickly read the text in order to locate explicit pieces of information scattered through the whole text/paragraph, participants most frequently had to rapidly read the entire text (S2) in order to locate any piece of necessary information to the completion of the item task. Since the pieces of information necessary to answer these test questions were scattered in different parts of the text, expeditious reading of the entire text was not sufficient in itself. Some examinees supplemented it with a careful reading of a portion of the text (S3) generally conducted after expeditious reading of the whole text. This type of reading aimed to search any useful information located in a specific paragraph and that could contribute to answering the question. Some examinees conducted careful reading of the whole text (S1). Since examinees could not predict the location of information necessary for answering this type of items, and since they could not anticipate the form the needed information could take, some participants found it important to augment their text understanding by using their knowledge of grammar (S5), a strategy that could aid them to parse sentences and construct an appropriate comprehension of specific piece of information located in a specific part of the text. Since test items that target this skill require examinees' ability to locate scattered pieces of text information by reading both expeditiously and carefully at global level, such test questions may appear to be difficult for non-skilled readers. This result is confirmed by the low mean item difficulty index (.4) that suggests the seven test items were generally difficult.

\section{Task Complexity for Expeditious Reading at Local Level Test Items}

The information contained in Table 1 confirms that in order to answer these items, three main strategies were used by participants: search for the part of the text recommended (S8) [for example: "The contrary of 'hardly' underlined in the last paragraph is:"]); then carefully read that text portion (S3) in order to either use clues provided by the context to understand the word, or parse the sentence in order to understand the actual use of the grammar structure. In some cases, participants had to resort to their knowledge of grammar (S5) as well as their knowledge of vocabulary (S6) to aid comprehension. Besides, in order to answer these test questions, participants mainly selected the answer through elimination of all implausible options (S12), or they produced the answer immediately after reading the question, and they could look at the five options only to confirm the answer (S11). The use of these strategies suggests that this type of test items may be relatively easy for examinees. This result is not supported by the mean item difficulty (.5) that indicates that the six test items included in this category were of moderate difficulty.

\section{DISCUSSION}

The statistics presented in Table 2 suggest that ESE test constructors put more focus on careful reading (64\% of test items) than on expeditious reading (36\% of test items). Strategies use indicates that careful reading items appear to require a frequent combination of strategies for reading carefully and expeditiously at both global and local level; while expeditious reading items appear to require less frequent combination of strategies for reading carefully and expeditiously at both global and local level. This finding suggests that tasks that target careful reading may appear more complex for examinees than those that target expeditious reading. This result is supported in mean item difficulty index (.5 and .6 for careful reading and expeditious reading respectively). However, since the DR Context supports that reading is conducted almost exclusively in classroom with little or almost no reading supports, ESE tasks appear to be inappropriate as there is preponderance of tasks that target careful reading over those that target expeditious reading. The ESE may need some modifications to more closely reflect the actual context of students by including tasks that test both careful reading and expeditious reading in a fairly balanced proportion.

The information in Table 3 suggests that the majority of test items target reading at global level (56\% of test items) than reading at local level (44\% of test items). Yet, test tasks that target global level appear to be more difficult (ID=.5) than those that targeted local level (ID=.6). This finding is in accordance with findings reported by Rouet et al. (2001), van Steensel, Oostdam \& van Gelderen (2012) and Song (2008). The preponderance of test tasks that target global level over those that target local level does not reflect the context of the DR Congo English state examination characterized by a paucity of reading resources, low students' motivation in English, some negative attitude towards the ESE, etc. Therefore, the test needs some revision to include more 'low-level questions' as these questions mainly require examinees to read the text at local level by focusing on text micro-propositions rather than 'high-level questions' as these mainly require examinees to read the text at global level by focusing on text macro-structure and by attempting to generate more connections between their knowledge and text information.

In many cases, careful reading was found to be used in conjunction with expeditious reading; especially with questions that target careful reading of a portion of the text as examinees had to first read the whole text (either carefully or expeditiously, or both) in order to integrate the paragraph macrostructure into the overall text gist. This combination of careful reading and expeditious reading suggests that reading activity may not be necessarily linear and these two types of reading may involve some overlapping processes and actions.

Finally, the results also suggest that reading at global level may be seen as a general strategy used for answering even some test items that target information at sentence level. Therefore, global reading might aid struggling readers to build text gist necessary to understand local pieces of information.

\section{CONCLUSION}


Validity is about the meanings and appropriateness of test scores (Messick, 1989). In order to construct a valid multiple-choice reading test, the test constructor must ensure that the test includes tasks that are appropriate to examinees' characteristics in such a way that they can deploy strategies that reflect the fact that they have been actually actively working to understand the text, to understand the expectations of the questions, to understand the meaning and implications of the different item options in light of the text, and to select and discard options based on the way they understand the text. When test tasks are beyond examinees' reading level, inferences and decisions taken on the basis of test scores may not be relevant as they may not provide a clear indication of examinees' reading skills.

\section{APPENDIX: STRATEGY QUESTIONNAIRE}

Instructions:

- You will have 2 hours to do the test and fill out the questionnaire.

- Read the text and provide answers to questions based on the text.

- After answering each test question, please fill out the questionnaire for that question. To fill out the questionnaire, you will have to identify and list in order ALL the strategies you remembered you have used to answer that individual question. The list of strategies and their code are present in the table below.

- For example, if to question 1 you first read the text carefully, and then reread it rapidly and finally look at the question options and produce your own answer after reading the question, you must write: S1; S4; S11.

\begin{tabular}{|l|l|}
\hline Code & Strategy \\
\hline S1 & read the whole text carefully \\
\hline S2 & read the whole text rapidly \\
\hline S3 & read a portion of the text carefully \\
\hline S4 & read a portion of the text rapidly \\
\hline S5 & use my knowledge of grammar \\
\hline S6 & use my knowledge of vocabulary \\
\hline S7 & draw conclusion based on what the text implies \\
\hline S8 & search for part of the text recommended \\
\hline S9 & consider the options and postpone consideration of the 'correct' option \\
\hline S10 & produce my own answer after reading the question, then look at the options \\
\hline S11 & select option through elimination of other options \\
\hline S12 & select option through guessing \\
\hline S13 &
\end{tabular}

\section{REFERENCES}

[1] Bernhardt, E.B. (1991). Reading development in a second language. Norwood, NJ: Ablex.

[2] Cerdan, R., Vidal-Arbarca, E, Martinez, T., \& Gil, L. (2009). Impact of question-answering tasks on search processes and reading comprehension. Learning and Instruction, 19 (1), 13-27.

[3] Rouet, J. F., Vidal-Abarca, E., Erboul, A. B., \& Miligo, V. (2001). Effect of information search tasks on the comprehension of instructional text. Discourse Processes, 31 (2), 163-186.

[4] Cohen, A. \& Upton, T. (2007). I want to go back to the text: Response strategies on the reading subtest of the new TOEFL. Language Testing, 24 (2), 209-250.

[5] Enright, M., Grabe, W., Mosenthal, P., Mulcahy-Ernt, P., \& Schedl, M. (2000). A TOEFL 2000 framework for testing reading comprehension: A working paper. Princeton, NJ: Educational Testing Services.

[6] Ericsson, K. A., \& Simon, H. A. (1993). Protocol analysis: Verbal reports as data, Cambridge, MA: MIT Press.

[7] Faerch, C., \& Kasper, G. (1987). From product to process - introspective methods in second language research. In F. Faerch and G. Kasper (Eds.), Introspection in second language research (pp. 5-23). Clevedon: Multilingual Matters Ltd.

[8] Grabe, W. (1999). Developments in reading research and their implication for computer-adaptive reading assessment. In M. Chalhoub-deVille (Ed.), Issues in computer-adaptive testing of reading proficiency (Studies in Language Testing 10) (pp. 1147), Cambridge: Cambridge University Press.

[9] Hirano, K. (2009). Research on test-taking strategies in L2 reading. Bull. Joetsu Univ. Educ. 28, 157-165.

[10] Hoover, W., \& Tunmer, W. (1993). The components of reading, in G. Thompson, W. Tunmer, \& T. Nicholson (eds.), Reading acquisition processes (pp. 1-19), Clevedon, England: Multilingual Matters Ltd.

[11] Katalayi, G.B. (2011). The DR Congo English state examination: Some fundamental validity issues (Context validity evidence), Unpublished Master Thesis, Department of Language Education, Faculty of Education, University of Western Cape, South Africa.

[12] Khalifa, H., \& Weir, C.J. (2009). Examining Reading: Research and practice in assessing second language reading, Studies in Language Testing 29, Cambridge: UCLES/Cambridge University Press.

[13] Kuusela, H., \& Paul, P. (2000). A comparison of concurrent and retrospective verbal protocol analysis. American Journal of Psychology 113 (3), 387-404.

[14] Levine, A., Ferenz, O., \& Reves, T. (2000). EFL academic reading and modern technology: How can we turn our students into independent critical readers? TESL EJ, 4 (4), 75-91.

[15] Mebarki, Z. (2011). Factors underlying the reading performance of Algerian microbiology students. Procedia- Social and Behavioural Sciences 29, 1759-1768. 
[16] Messick, S. (1989). Validity. In R. L. Linn (Ed.), Educational measurement (3rd ed., pp 13-103). New York: American Council on Education.

[17] Munby, J. (1978). Communicative syllabus design. Cambridge: Cambridge University Press.

[18] Nevo, N. (1989). Test-taking strategies on a multiple-choice test of reading comprehension. Language Testing, 6 (2), $199-215$.

[19] OECD, (2003). The PISA 2003 assessment framework: Mathematics, reading, science and problem solving knowledge and skills. Paris: OECD.

[20] Park, S. (2009). Verbal report in language testing. The Journal of Kanda University of International Studies, 2, $287-307$.

[21] Purpura, J. (1998). Investigating the effects of strategy use and second language test performance with high and low-ability test-takers: a structural equation modeling approach. Language Testing, 15 (3), 339-379.

[22] Rayner, K., \& Pollatsek, A. (1989). The psychology of reading. Englewood Cliffs, NJ: Prentice Hall.

[23] Rouet, J. F., Vidal-Abarca, E., Erboul, A. B., \& Miligo, V. (2001). Effect of information search tasks on the comprehension of instructional text. Discourse Processes, 31 (2), 163-186.

[24] Rupp, A., Ferne, T., \& Choi, H. (2006). How assessing reading comprehension with multiple-choice questions shapes the construct: a cognitive processing perspective. Language Testing, 23 (4), 441-474.

[25] Sarojani, K. \& Krishnan, D. (2011). Careful versus expeditious reading: The case of IELTS reading test. Academic Research International, 1 (3), 25-35.

[26] Sasaki, M. (2000). Effects of cultural schemata on students' test-taking processes: a multiple data source approach. Language Testing, 17 (3), 85-114.

[27] Song, M. Y. (2008). Do divisible subskills exist in second language (L2) comprehension? A structural equation modeling approach. Language Testing, 25 (4), 435-464.

[28] Urquhart, A. S. \& Weir, C. J. (1998). Reading in a second language: Process, product and practice. Essex: Pearson Education Ltd.

[29] Van Steensel, R., Oostdam, R., \& van Gelderen, A. (2013). Assessing reading comprehension in adolescent low achievers: Subskills identification and task specificity. Language Testing, 30 (1), 3-21.

[30] Weir, C.J., \& Khalifa, H. (2008a). A cognitive processing approach towards defining reading comprehension, Cambridge ESOL: Research Notes, 31, 2-10.

[31] Weir, C.J., \& Khalifa, H. (2008b). Applying a cognitive processing model to Main Suite reading papers, Cambridge ESOL: Research Notes 31, 11-16.

[32] Weir, C. J. (2005). Language testing and validation: an evidence-based approach. Palgrave: Macmillan.

[33] Weir, C., Hawkey, R., Green, A., \& Devi, S. (2008). The cognitive processes underlying the academic reading construct as measured by IELTS. IELTS Research Reports, 9, 157-189.

[34] Weir, C. J., Hawkey, R., Green, A., Unaldi, A., \& Devi, S. (2008). The relationship between the academic reading construct as measured by IELTS and the reading experiences of students in their first year of study at a British university. IELTS Research Reports, 9, 97-156.

[35] Weir, C. J., Yang, H., \& Jin, Y. (2000). An empirical investigation of the componentiality of L2 reading in English for academic purposes. Cambridge: Cambridge University Press.

[36] Yamashita, J. (2003). Processes of taking a gap-filling test: comparison of skilled and less skilled EFL readers. Language Testing, 20 (3), 267-293.

Godefroid B. Katalayi is a PhD Candidate in Language Education, Faculty of Education, University of the Western Cape, South Africa. He is also Senior Lecturer at "Institut Superieur Pedagogique de Kananga", Democratic Republic of the Congo where he lectures Language testing and English teaching modules at the Department of English. His research interest includes test validity and the teaching of reading.

Sivakumar Sivasubramaniam is Associate Professor and Head of the Language Education Department in the Faculty of Education at the University of the Western Cape, Republic of South Africa (RSA). He also serves the Editorial Board of the Journal of English as an International Language (EILJ) as Chief Editor and the Editorial Board of Asian EFL Journal (AEJ) as Associate Editor. He has been a foreign language/ second language educator for over thirty years now and has taught English in India, Ethiopia, Thailand, Bahrain, Armenia, and U.A.E prior to relocating to the Western Cape. He holds an MA in English Literature from the University of Madras, India, an MA in (Linguistics) TESOL from the University of Surrey, U.K and a PhD in Applied Linguistics from the University of Nottingham, U.K. His research interests include response-centred reading/ writing pedagogies, literaturebased language pedagogies, constructivism in EIL, second language advocacy, narratives in language education and text-based approaches to academic and social literacy practices. 